\title{
Nanoscale Examination of Biological Tissues Using X-ray Spectromicroscopy
}

James Everett ${ }^{1,2 *}$, Vindy Tjendana Tjhin ${ }^{2}$, Jake Brooks ${ }^{2}$, Frederik Lermyte ${ }^{2}$, Ian Hands-Portman ${ }^{3}$, Jon Dobson $^{4,5}$, Joanna Collingwood ${ }^{2}$ and Neil Telling ${ }^{1}$

${ }^{1 .}$ Institute for Science and Technology in Medicine, Keele University, Stoke-on-Trent, UK

2. Warwick Engineering in Biomedicine, School of Engineering, University of Warwick, Coventry, UK

3. School of Life Sciences, Gibbet Hill Campus, University of Warwick, Coventry, UK

4. Department of Materials Science and Engineering, University of Florida, Gainesville, USA

5. J. Crayton Pruitt Family Department of Biomedical Engineering, Institute for Cell and Tissue Science \& Engineering, University of Florida, Gainesville, USA

* Corresponding author, email: j.everett@ keele.ac.uk

Establishing the composition and distribution of chemical compounds within biological materials is fundamental to understanding the chemistry that drives life. Additionally, region-specific changes in chemical homeostasis have been linked to disease states [1]. Traditional techniques used to explore these relationships rely on the use of fixatives and dyes, which can significantly alter the native chemistry of the sample material [2]. Here we describe methodology for the preparation and examination of biological sample materials, including human tissues, using the X-ray Spectromicroscopy technique Scanning Transmission X-ray Microscopy (STXM). Through STXM we show the distribution and speciation of organic and inorganic materials within biological samples at a nanoscale resolution. Importantly this approach does not require the use of dyes, aldehyde fixatives or contrast agents and therefore offers an unprecedented insight into the native chemistry of biological samples.

Human brain tissues were ethanol dehydrated and embedded in a resin comprised of equimolar trimethylolpropane triglycidyl ether: 4,4'-methylenebis (2-methylcyclohexylamine). Semi-thin (typically $200-500 \mathrm{~nm}$ ) sections from embedded samples were cut using a non-metallic blade, before being mounted onto a support. STXM was performed at the carbon $K$-edge, oxygen $K$-edge, calcium $L$-edge and iron $L$ edge. To map distributions of chemical species, paired images were taken at the energy corresponding to a peak feature of interest and an off-peak energy a few $\mathrm{eV}$ away from this feature. The off-peak image is then subtracted from the peak image to give a difference map. This process is demonstrated in Figure 1, for a $200 \mathrm{~nm}$ thick section of human substantia nigra tissue imaged at the carbon $K$-edge. Here the peak image (288.3 eV; left) corresponds to the $1 \mathrm{~s}$ to $\pi^{*}$ transition for amide groups (proteins), whereas the offpeak image ( $290 \mathrm{eV}$; middle) corresponds to the principal carbon $K$-edge absorption feature for the resin (see also [1]). This image processing allows artifacts and background resin absorption features to be removed, revealing the true tissue structure. From these difference maps, tissue structure is shown to be well preserved over the $160 \mu \mathrm{m}^{2}$ area presented. The high resolution map taken at ca. $40 \mathrm{~nm}$ resolution (right; inset) shows cellular structures, also displaying subcellular organelles.

Further speciation maps and a carbon $K$-edge absorption spectrum from a $200 \mathrm{~nm}$ thick section of human amygdala tissue are shown in Figure 2. X-ray absorption spectra were obtained from a series of images taken at energies spanning the carbon $K$-edge edge. The resulting spectrum from tissue area A1 (Figure 2f; green spectrum) was consistent with a synthetic peptide standard (Figure 2f; black spectrum), indicating the tissue to have been preserved through the embedding process. Further dense areas of tissue (as evidenced by enhanced contrast in Figure 2a) were found to be loaded with calcium (Figure 2b), carbonates (Figure 2c) and iron (Figure 2d). This demonstrates STXM to be sensitive to altered chemical 
compositions throughout tissue sections. These findings validate the use of X-ray spectromicroscopy for the examination of biological materials, including tissues of human origin, demonstrating these techniques to be suitable for determining (nanoscale) regional differences in chemical composition.
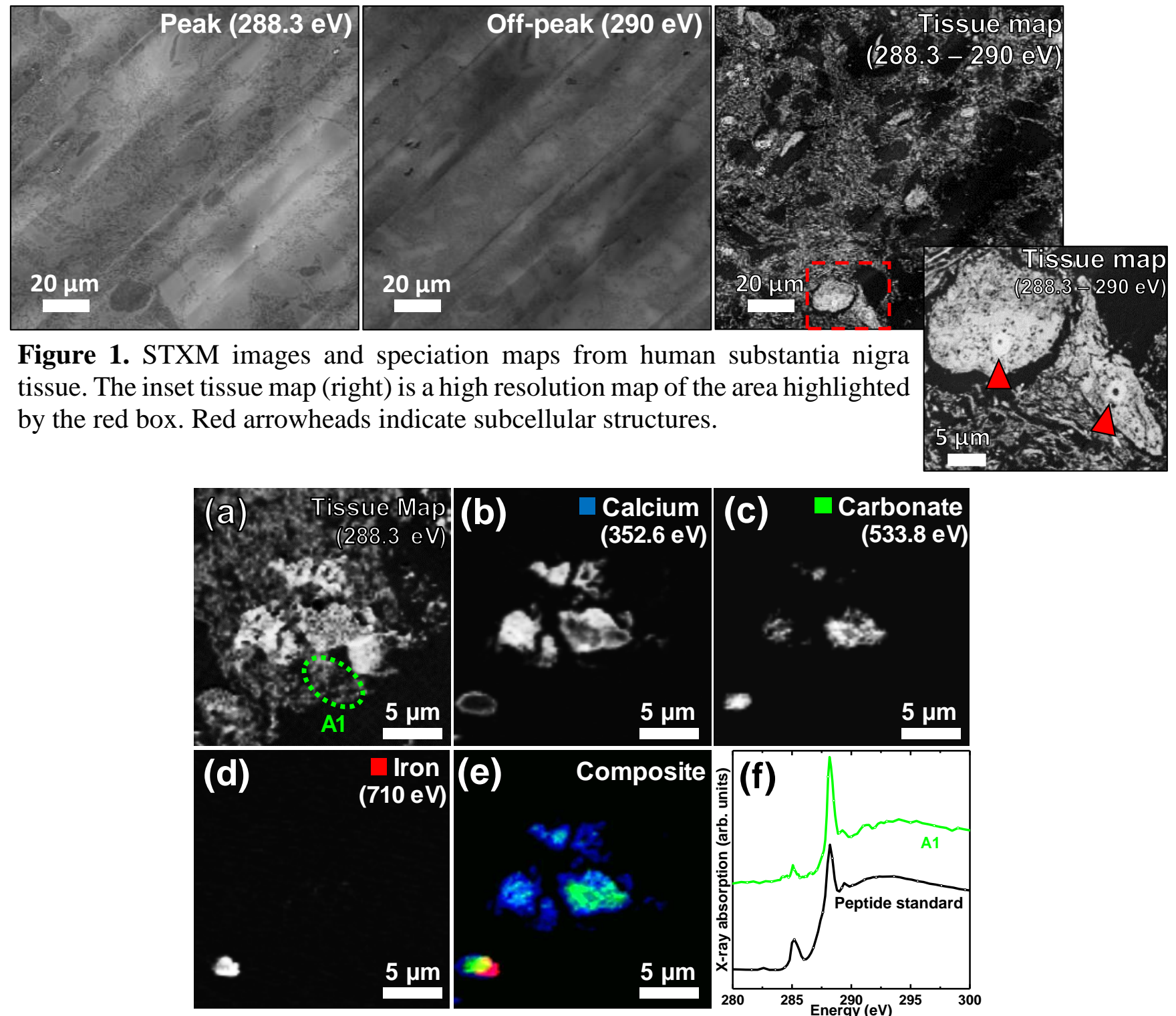

Figure 2. (a)-(e) STXM speciation maps, (f) carbon $K$-edge absorption spectrum from amygdala tissue.

\section{References:}

[1] N D Telling et al. Cell Chem. Biol. 24 (2017), 1205-1215

[2] J A Kiernan "Histological and Histochemical Methods" ed. 5 (Scion Publishing, Banbury, UK)

[3] This work was supported by EPSRC grants EP/K035193/1, EP/N033191/1 - EP/N033140/1 and University of Warwick alumni donations. Tissues were obtained with informed consent, and analyzed in accordance with the Declaration of Helsinki under the remit of ethical approval 07/MRE08/12 from the UK National Research Ethics Service. We thank staff at the Advanced Light Source beamline 11.0.2 and Diamond Light Source beamline I08 for assistance with data collection. 